\title{
Increasing Obesity in Ugandan Women due to Transition from Rural to Urban Living Conditions? a Qualitative Study on Traditional Body Image, Changed Lifestyles and Unawareness of Risk for Heart Disease
}

\author{
Ellis Janzon ${ }^{1}$, Sanyu Namusaazi ${ }^{2}$ and Ingrid Bolmsjö ${ }^{3}$ \\ ${ }^{1}$ Health and Society, Malmö University, Malmö, Sweden \\ ${ }^{2}$ Uganda Media Women's Association, Kampala, Uganda \\ ${ }^{3}$ Ellis Janzon, Malmö University, Health and Society, Institution of Welfare Studies, \\ Jan Waldenstromsgatan Malmö, Sweden
}

Correspondence should be addressed to: Ellis Janzon; ellis.janzon@mah.se

Received date: 29 December 2013; Accepted date: 10 April 2014; Published date: 2 February 2015

Academic Editor: Montserrat Bacardi-Gascon

Copyright @ $\odot$ 2015. Ellis Janzon, Sanyu Namusaazi and Ingrid Bolmsjö. Distributed under Creative Commons CC-BY 4.0

\begin{abstract}
Globally, heart disease is still a major public health problem. In developed countries, this is due to an ageing population, and in developing countries, like Uganda, it is due to increasing negative lifestyles. Many women in Uganda move from rural to urban areas, thereby changing their diet, reducing their level of physical activity, and increasing their weight as well as their risk of myocardial infarction. Method The qualitative method was used in this study. Twelve women aged over 40 years, who within 10 years had moved from the countryside, into the city of Kampala, were interviewed by the use of a semi-structured question guide. Data were analyzed by means of Burnard's content analysis method and by using the Theory of Reasoned Action.The aim was to examine women's experiences and perceptions of body image, lifestyles and overweight in relation to a change of living conditions, and, furthermore, to examine their knowledge of the risks of heart disease. The results showed the following themes: body image, overweight, physical inactivity, culture, transition, women's needs, concerns and lack of knowledge about the risk of heart disease. The core finding was that in spite of an income increase, a reduced health situation was described. All the interviewees reported increased body weight, encouraged by their surroundings. Lack of knowledge about nutrition and risk factors for heart disease, i.e., overweight and inactivity, and the fact that being obese was perceived, by themselves and others, as beautiful, were reported. Conclusion Important knowledge gained for public health measures was that interventions increasing women's heart health should be geared towards women's empowerment. Strategies ought to raise knowledge and awareness of forces and structures in Ugandan women's surroundings that keep them inactive and make them increase their body weight. It is important to give the women support in taking charge of their own body and health.
\end{abstract}

Keywords: attitudes, body image, lifestyles, knowledge of heart disease, transition

Cite this Article as: Ellis Janzon, Sanyu Namusaazi and Ingrid Bolmsjö (2015),"Increasing Obesity in Ugandan Women due to Transition from Rural to Urban Living Conditions? a Qualitative Study on Traditional Body Image, Changed Lifestyles and Unawareness of Risk for Heart Disease", Journal of Research in Obesity, Vol. 2015 (2015), Article ID 213083, DOI: 10.5171/2015.213083 


\section{Background}

In Uganda, increasing rates of heart disease have been shown the last decade, probably due to a shift away from Uganda's traditional way of life. The city of Kampala has grown rapidly in

population the latest years (Uganda Bureau of Statistics, 2006). The country has seen a positive shift from less poverty and undernutrition, and its related diseases, to an increase in chronic non-communicable diseases such as cardiovascular diseases (CVD), followed by a $6 \%$ increase of all medical admissions, as well as related risk factors, i.e., diabetes, hypertension, and obesity, among others (Atkinbooy et al, 2003; Schenk-Gustavsson, 2009). Most of the women that migrate from urban settings do so due to poverty and search for jobs. They are also, for the most part, illiterate and consequently get the most badly paid jobs (Uganda Bureau of Statistics, 2006). After migration, they earn a salary, which, though it might be low, enables them to make a change in lifestyle that might reduce their health status. Before migrating, they consumed traditional low-calorie and unprocessed food, but after migrating, they change to a more Western diet of more processed and fried fast food. At the same time they also reduce their level of physical activity by using bikes or public transportation instead of walking long distances. As a result, many of these women experience overweight.

Overweight is a well-known risk factor for heart disease and for, for example, diabetes and high blood pressure. In Uganda, women still have a traditional family role, taking all the responsibility for the home, the children, and elderly relatives, besides their salary-paid work. Thereby, they might experience increasing stress levels and reduced possibilities to prioritize their own health and well-being. In Uganda, as in any developing country, there are few possibilities to offer the population healthprevention measures, including early detection services for heart diseases. Processed food is becoming common habits and its negative health consequeses have been expressed in the National Health Sector Plan (Uganda Ministry of Public Health, 2005). For public health, it is an important issue and a challenge to tackle getting the population interested in physical activity and in diet modification. Women in Uganda do not share the Western societies' perception that women ought to be slim (Martorell et al, 2000). Their perception is that a thin/slender female form looks bad, and they believe that being obese is a sign of being healthy and attractive, a belief in which they are supported by partners and surroundings (Martorell et al, 2000; Coetzee \& David, 2011).

Preventable well-known risk factors for CVD are, among others, dyslipidaemia, hypertension, smoking, stress, diabetes, obesity, physical inactivity as well as a poor diet. Some risk factors are even synergic, such as smoking in combination with high blood pressure (Janzon et al, 2004)). Many of these risk factors can be reduced by lifestyle modifications, such as increased physical activity and diet modification (Janzon et al, 2004; Haskell et al, 2007). MI kills more women each year than any other disease (Schenk-Gustavsson, 2009).

All persons live their bodies and have their own picture of it. This incorporates the woman's attitude to her body as well as the perception that it is an "expression" of her sexuality

(Buss \& Schnitt, 2011).

The feminist theorists argue that women often adopt the observer's attitudes to and perceptions of their bodies (Buss \& Schnitt, 2011). What forms the experience of women's body image are factors like the norms of the social context, the culture, and the perceptions of "significant others". Everybody is affected by the norms of their culture about the mean "ideal body" image, presented in different media, such as newspapers, TV, or films, i.e., about how the perfect body appearance of a woman ought to be in order to be seen as attractive in the eyes of others. Her body image is valued above all by possible sexual partners evaluating her possible sexual 
attractiveness, fertility, and disease resistance, thereby perceiving her as attractive/beautiful or not (Coetze, 2011). African men often prefer a significantly larger female body size and are more willing to date women who conform to that (Buss \& Schmitt, 2011). Heavier women in some African cultures are evaluated as highly attractive and respectable. This affects the African women's perception of their own bodies, they consequently eat more and have a higher BMI (Buss \& Schnitt, 2011; Barky, 1990).

On that background, women in Uganda do not often engage in dieting or physical activity for body control (ByrdBredbrenner \& Murray, 2003). They perceive thinness as a sign of poor health or low economic circumstances (Stevens et al,1994). This perception of body size may lead many African women to underestimate their risks for heart disease. Studies show that women are often afraid of getting cancer more than anything else and that they are unaware of the risk of heart disease (Padgett \& Biro, 2003). Nevertheless, increasing weight can be dealt with by increased physical activity as well as by diet restriction, but many middle-aged African women may think that physical activity is not appropriate for them or meant only for young people.

On this background, it was found important to further explore what women think about their own risk of heart disease in relation to their transition from a rural lifestyle to an urban living with changes in lifestyle, and hopefully hereby gain knowledge for creating public health measures for women's heart health.

\section{Aim}

The aim of this study was to examine women's experiences and perceptions of body image, lifestyles, and overweight in relation to a change of living circumstances from a rural to an urban lifestyle. The aim was, furthermore, to examine the women's knowledge of the risks of heart disease.

\section{Method}

When the purpose of a study is to get a deeper understanding of feelings and attitudes, a qualitative method is appropriate. In this study, interviews were carried out. The question guide was designed to obtain women's perceptions of body image, their lifestyles and knowledge of risks of heart disease.

\section{Inclusion Criteria}

The women in the study had, within the last ten years, moved from the countryside into the city of Kampala, Uganda, to find work. They were interviewed in their homes, at workplaces, or in their church, depending on where they felt it was convenient. Those women that fitted inclusion criteria were contacted through the church. After the end of each interview the respondent was asked if she was aware of another woman who like herself had moved to the city within the latest 10 years and, if so, if she would put us in touch with that woman.

\section{Informants}

The women were interviewed using a designed questionnaire guide with semistructured interviews, targeting women aged 40 years and above. Twelve women were interviewed who all had a work occupation, only one of them had a higher education, all others worked with low or none educational jobs within office or trade. After having interviewed the 12 women, no new information was added in relation to the aim, since saturation in the interviews seemed to have been accomplished.

\section{Collecting and Analyzing the Data}

The findings were collected by one of the researchers, transcribed and analyzed in a logical order that focused on the meaning and linguistic form of the text that plotted the respondents' stories. All interviews were read and discussed in the scientific group in order to share knowledge and understanding and find themes, according to qualitative methods described by Kvale (2009). 
The results were analyzed by using Burnard's (1991) content analysis method and by the theory of Reasoned Action by Ajzden and Fishbein (1980). The data were thereafter coded in an understandable way, which provided a framework for thematic ideas (Kvale, 2009). The analyzing process was based on the twelve stages described by Burnard (1991) that continuously encourage the researcher to purposely explore transcripts and those aspects mentioned in the text by the women respondents in the interview. The coding of the transcripts was then read again line by line and in that way the themes emerged and were categorized. In the next stage, the categories were put into broader themes as headings. The headings were read thoroughly and all the coded items were given the understanding of the phenomenon and they were found to represent the voice of interviewed respondents. After this procedure, the writing process began. Through this process, the researcher was able to capture new insights for prompt further investigation as well as selecting the data for future interviews (Kvale, 2009; Burnard, 1991; Silverman, 2006).

\section{The Interviews}

The interviews were carried out in an environment where women lived their daily life, i.e., work places, homes, and churches, using a purposive snowball technique. The first respondent was contacted through a local church in Kampala, Uganda. After the end of the interview, each respondent was asked if she knew of any other women with the same background fitting the aim of the study, and in that case, would she help to make contact with those women. This method called "the Snowball" data collection method is often used in qualitative studies (Kvale, 2009), and such a "data catching" method is often used by other scientists and is regarded as a constructive way to capture events in the life worlds of the respondents as they unfold (Kvale, 1991; Silverman, 2006). The interview guide was semi structured and during the study it guided the interviewer, who was thus able to capture emerging ideas, attitudes, and unexpected information from the informants (Silverman, 2006). The interviews were conducted with one and the same interviewer (in the local language Luganda or in English, according to the preference of the respondent). The intervjuar was trained in intervju tecnic from before and was educated in qualitative methods. The themes of the interviews mainly followed the aim of this study as stated in the question guide and they were answered during the interviews. The informants were encouraged to speak in their own words, and the researcher endeavored to limit the 'Yes' or 'No' answers, and sometimes raised additional questions for clarity and expansion on what was said. The interviews were recorded not to loose any important information and in order to make it possible to later play back and listen to the tape at the time of transcribing in data analysis (Kvale, 2009). The interviewer sometimes also took notes during the interviews.

The interviews were transcribed as soon as possible into a Word-document, following the interview guide as a frame for the text. The hand-written notes from the interviews were also thoroughly read, since these notes were an extra support to the findings; this was the case if some part of the tape was hard to hear, or was not understood.The transcription of the findings was done by selecting important parts relevant to the study, whereby the researchers tried to condense the interviews and organized them in a logical manner in order to attribute meaning to and gain understanding of the answers of the interviewees. Answers that were given in Lugande were also translated into English language.

All participants gave signed consent before the interviews. Furthermore, information about the study aim and data security was given to all informants orally and in writing before the interviews. If the respondent could not read, the information was read or explained to them. They were also guaranteed that no names would be mentioned anywhere in any documents. The study was approved by The Ethics 
Board of Lund University and The Ethics Board of Malmö University, Health and Society.

\section{Findings}

After data analysis of the interviews, the following themes emerged: "Body image", "Culture", "Knowledge", "Transition", "Women's needs", and "Concerns about disease".

\section{Body Image}

There were mixed feelings about the question of one's "own body image" among the respondents. Not all the women were happy about their weight increase; one of them felt shapeless, and another used to be proud of her slender model-like body, but another did not worry about it.

"My body appearance - I don't like it, because of my tummy. And I feel I am not beautiful with my shape, I feel my figure is shapeless. And I need help in one way or the other ..." (Participant 3)

"Before, I used to be a slender girl and I was very proud of my body form because I took myself as a model by that time. But later my life changed a lot." (Participant 1)

While Participant 6 said: “OK! But people tell me to reduce my weight, but me I feel oK."

\section{Culture}

All women communicated that being thin meant loss of beauty, since in the African context men prefer big size women, perceiving them as beautiful, attractive, sexy, and respectable. Obese women are thereby more eligible for marriage and would give the man more status in relation to his family.

“... people think about body ideals in connection with marriage. Normally men want women who are fat, they think these are of integrity and respect, despite other problems. And still think that the more weight one puts on, the more beautiful and attractive. Women still want to be of big size rather than being slim, slender women. The ideal behind this is that fat men get attracted to fat women more than to slender ones, that fat women look beautiful and are attractive ..."

(Participant 2 and 8)

“... in marriage relationships, the family you are marrying into, will kind of belittle you because of being of a small size. So in most cases that's why even a man you are marrying tries his level best to see to it that his wife puts on more weight in light of being proud of his wife and respect ..." (Participant 3)

Two respondents stated that their husbands got worried if they lost weight and rather supported them in keeping their weight.

“... my husband doesn't comment on my weight, he likes me being fat! And when he realizes that I have lost weight, he comments and get concerned, meaning he doesn't want me to lose weight..." (Participant 1)

Similarly, some women stated that being thin or slender would be a sign of not doing well and being unhealthy.

“... In the context of culture, people still think the more weight you gain and become fat, that's being attractive to the husband and gaining respect in the eyes of the relatives of the family you intend to marry into. ... because if the son for instance marries a woman who is slim, they think that the woman is HIV positive. So they prefer their son to get married to a fat woman who earns him and the family respect. And if a woman is thin, they think she has problems, thus thinking that the more weight she has the healthier she is ......"

(Participant 2)

In addition to being healthier one of the respondents stated that

“......When I gained more weight some people started commenting whereas a few were saying it's $O K$ ! The fact that my husband doesn't comment on my size makes me feel comfortable. Most men prefer having fat women rather than slender women. So I can conclude that the surroundings do not 
mind about my size as long as my husband accepts it. Because me, I was so much worried about my husband commenting on $i t$, but then he is not the type of person who is bothered about my size, being fat." (Participant 2)

Overweight was demonstrated to influence women's body image and health status, as they were perceived as wealthy. The result of gaining more weight was a scenario described again in the statements of a woman respondent: "...You know in Uganda people go by someone's looks. People normally say I look nice and beautiful. And I feel comfortable with my weight. You know when you lose weight people tend to think that you have problems. So I have to maintain this weight. People think that with my weight I have money and am doing well in life and of course this weight earns me some respect compared to slim and slender women ...(Participant 2)

\section{Knowledge}

Some of the women lacked both knowledge about, and interest in, heart disease and the positive health effect of physical activity. Lack of education about heart disease was expressed as a hindrance to the effective management and reduction of overweight. They also commented that even in cases of existing knowledge, other perceptions were dominating women's behavior. Only rarely had the women ever got any health advice concerning overweight or a sedentary lifestyle. However, one of the women did mention that she had had advice about losing weight and exercise.

“... of late I went to Europe and I learnt a lot that doing exercise is good for our health. Because in Europe it's part of their life. This motivated me a lot. I started doing some exercise slowly by slowly since I was not used to such and I was not taking it very seriously while in Uganda, but while in Europe I had to set myself doing it. Though I acquired some knowledge, am still not taking it so seriously ..." (Participant 1)

"... today such knowledge is not taken into consideration, it's only those who have interest in it,...
... I also started changing my diet. These days I do not take fatty foods and oils, and at least I try to prepare vegetables and sometimes fruits ..." (Participant 1)

“... In Uganda the problem is that we rarely get such advice concerning the risks of heart disease, so I think there is a gap which we have to fill ..." (Participant 7)

Several of the women explained that even when they sometimes went to the hospital and were weighed by health professionals, they were seldom informed about the health risk of overweight or had any diet advice or recommendations about increasing physical activity. Additionally, one woman participant explained that

“... medical advisors normally weigh us only when we go to the hospital for treatment. Surprisingly they do not reveal anything concerning overweight." (Participant 3)

"... I have never had any advice concerning health promotion. The only thing they do that's when I go to hospital, they only weigh your weight, and record it, but they never advise you on how to promote your health." (Participant 1and 4). "So I think this is something good if this kind of intervention is implemented in the future. It will enlighten women's thinking about the risks of heart disease to improve on their health..." (Participant 1)

“...they never say anything about overweight, and the risks of being fat. So they are reluctant, maybe that's why we are also reluctant about noticing the issue of overweight ..." (Participant 2)

Furthermore, besides the lack of knowledge about overweight and the risk of heart disease that emerged as a worry for women who experienced symptoms of "heart insufficients", there also seemed to be a misconception about the expression "good food", the assumption being that it meant tasty instead of healthy and good quality food, as expressed by one of the women.

“... women are not aware of the problems associated with such. They only know that 
eating well is to eat fried food, and not aware of the advantages of doing exercise. And most importantly to take note of having food that will not cause any harm to them, like that processed foods, it's advisable to every woman to have so training on what to eat, in what quantities and how to prepare would also be good in line of this theme and what type of physical activity recommended to be physically fit..." (Participant 1)

One of the women reported health symptoms due to her overweight: “... Actually when I do some walking my heart start beating very fast. And I shared it with my friend, and she told me it's not good for my health. However, I have a fear of losing much weight which might make me not look nice." (Participant 2)

Additionally, the women's social networks sometimes gave them advice about health and physical activity.

"... Some of my friends do give me some advice but I don't take it seriously and I feel lazy to get engaged in physical activity, and many times it's difficult to decide on what to eat as something good for my health, because I eat what I want at a time..." (Participant 3)

\section{The Transition and Changes in Lifestyle}

The respondents explained that after their move into the city they changed their lifestyle, and their eating habits, since they now expected the food to taste better and they wanted the food preparation to be faster and easier. Since they now could afford it, they bought more processed food and saw it as a life improvement, and they experienced the effect of that change.

“...But later my life changed a lot, because I could afford to buy everything I wanted in my life and I started growing fat slowly by slowly..." (Participant 1)

“... I used to be a small size girl and very tall. But when I moved to Kampala city, after my studies, my life changed a lot. One thing is that when you move to Kampala, you definitely have different perspectives in line of having life of good quality compared to the one in the rural areas. So when I moved to the city of Kampala this motivated me to change my lifestyle. You know it just comes automatically just because of the earnings I get per month" (Participant 2)

“... after my university education, my lifestyle changed, in such a way that the type of food I used to eat while in the village, it's not the type of food that am eating. You know in our village area we used to eat different types of food and not fried as we do it in the city life.

And I think because of city lifestyle, I want to make life as easy as possible especially when it comes to the way I prepare my food, it's not the way I used to eat it in my village. The type of food we used to eat was not fried only boiled, and here in the city you cannot, you feel like the food is not tasty. So the type of food I eat has to be fried, with oil, fats, and sugar, because in town you cannot dare take tea without bread and butter, fried chicken, pork or meat, yet in the village if you happen to have it, it means you don't have to make it fried and used to eat green vegetables which are not fried, different types of food which are nutritious and recommended to eat." (Participant 3)

And some explained the consequence of this new lifestyle on body size:

“... My weight increase came naturally slowly by slowly. Of which before I used to think it's normal to gain weight. Now I am over 90 kilogram. And I think this has come as a result of eating ready-made food, like chips, roasted meat, and chicken, together with other types of food which are processed, because most of the time I am in the office, and I find myself buying ready-made food, and sometimes food which is full of fats and oils. And some drinks like beer or bottle of soda..."(Participant 3)

\section{Hindrance to Physical Activity}

The respondents expressed different reasons for not being physically active. It could be laziness, high cost, or, for some, lack of work and time. For one woman, the sports dress code was a problem since she felt it was wrong for her age. Another hindrance that was mentioned was that 
there actually was nowhere to go and exercise! Yet, another woman claimed that the lack of social support and not having a friend to accompany her was a hindrance.

“... one thing am just lazy, another thing it's not cost effective as I told you that going to sauna and the gym has financial implications. Maybe if one pays for me and after having a long working day seated in the office ... "(Participant 2)

“...You know am a busy woman, I tried to make a timetable but I rarely follow it. I have a busy schedule. When I move out from home, I only find it hard to go out and do something else. I come back late at night and very tired to engage in any physical activity, So throughout the day I rarely find time to participate in physical activity." ( Participant 3)

"...is the way I have to dress while doing physical activity. It really puts me off, and become lazy to participate in physical exercise, if I have to participate, it's about dressing code, and at my age I feel putting on trouser or shorts, I cannot, since am an old woman, used to put on traditional wear at my age.

So I think doing exercise, it's for the young ones or the youths unlike us who are above 40 years. And on top of that I feel like losing my dignity and respect because here in Kampala there are no particular areas for doing exercise." (Participant 4)

"... is to have some money on me, so that I can afford to go the gym. I love it.

And if it was near my home, then I will be going there every day." (Participant 2 and 9)

“... I think if I could have a colleague or a partner who can help and give me company, that will maybe motivate me to participate in physical activity. But since I don't have one to encourage me to do so, then I see no reason to do it because me I take it as having fun." (Participant 3)

\section{The Women's Own Concerns about Disease}

The women did have different heart symptoms due to their overweight. Some had noticed difficulties in climbing hills and another complained that her knees had started to trouble her. In spite of that, the women preferred to stay overweight in order to keep their beauty.

“... Before I used to walk without feeling any complication, but now I cannot climb hilly areas as I used to do it. Most times my heart beats at high speed and I feel so exhausted unlike before when I was a slender girl. And in most cases when I try to do some exercise, I feel tired, so soon my heart pumps at a very high speed, which I feel is not normal."(Participant 1)

“ ... Actually when I do some walking my heart starts beating very fast. And I shared it with my friend, and she told me it's not good for my health. And I am a bit worried. Even I feel pain in my legs. I cannot squat as I used to be, a funny sound comes out from my knees, and this interview reminds me to do something about my weight. However, I have a fear of losing much weight which might make me not look nice." (Participant 2)

\section{Locus of Control}

All the women were aware, and expressed that they knew that it was their own responsibility to take action in some way to keep healthy.

"I think it's one's own responsibility to start thinking along those lines and get to know the advantages of doing exercise. So it's one's responsibility towards improving one's health." (Participant 1)

“...You know it's my life. I think it has to start with me! Yes! Before thinking of someone else to avoid all unnecessary problems including excessive weight and heart disease for instance ..." ( Participant 2) 


\section{Women's Needs to Keep Their Health}

The women expressed different needs regarding what would make it easier for or other women to keep healthy. They wanted help to be introduced to physical activity and also expressed the large knowledge gap about the risk of heart disease and the effect that healthy food and exercise would have on their bodies. They asked for more education on diet.

“... I think it would be better if I can get someone to train me on how to become physically fit, by providing relevant information on how to do it and what does it take to keep me healthy to avoid such kinds of serious diseases like heart attack and gaining unnecessary weight." (Participant 1)

"...women are not aware of the problems associated with such. They only know that eating well is to eat fried food, and are not aware of the advantages of doing exercise. So if women could access such information, I think it would be good to improve on their health. So training on what to eat, in what quantities and how to prepare would also be good in light of this theme and what type of physical activity is recommended to be physically fit ..."(Participant 1)

“... I think it would be good if some kind of sensitization is carried out in ligh of these themes, for women to keep healthy ..."(Participant 3)

"...To me I think going to a sauna and the gym it's more appropriate though it has got some financial implications. Because in most cases when you go to a sauna you end up being relieved from all the days stress, and it burns all the "fats" that one has in the body..."(Participant 2)

"...I think, moving out on weekends for a dance basically on Saturdays to make body movements. And also to mix with friends, many times when I move out, I feel happy, all the stress goes away though the next morning I feel a bit weak. But it's something I feel like most, going out for a dance on weekends and mix with my friends and be happy." (Participant 1)
"Yeah! ... I would really want to know, do I have to live without sugar? I need some information on this ... And also on the importance of being physically active. This will be my driving force towards improving my health ..." (Participant 7)

However, during the interview, it was obvious that the women were unaware of the importance of being physically active and that it was increasing their risk of heart disease. Instead, they focused on their body image, on being fat as a way of being attractive and beautiful to their husbands. Still, at the end of the interview, they understood the need to be physically active to maintain health effects and the need for a lower weight, in order to avoid unnecessary risks of heart disease.

\section{Discussion}

\section{Validity, Transferability and Reliability}

After having conducted interviews with twelve women, no new information in relation to the aim of the study emerged and thereby saturation was met. To ensure validity, all interviews were recorded, and interview notes were obtained, also on observations, in order to produce a meaningful and accurate comprehensive representation and thus get a true picture and understanding of the phenomenon, with the possibility of weaknesses of one method being checked by another. So, the interview method was carried out through a combination of interviews, recordings, interaction, observations, and notes. Since the sample was small, the findings cannot be representative demographically and for all women. Rather, transferability here was met by analytical generalizations. The knowledge gained can be related to the aim of this study and to public health concerns. Therefore, this study is applicable to all similar situations, questions and problems, regardless of demographic variables.

To deal with the issue of reliability, all interviews were conducted by one and the same interviewer. Also, the snowball technique employed in sampling was continuously modified, for instance, by searching for women fitting inclusion criteria. 


\section{Result Discussion}

The checklist to the guide contained leading questions with some of the questions having the same central idea but different phrasing, with the intention of testing the consistency of the responses from the interviewees.

A large group of men and women have been moving into the city of Kampala in search of jobs and better lives. They were employed in different offices, shops, or factories depending on their qualifications. The correlation between socio-economic status and health status is well established, the general pattern being that the higher the income the lower the risk of disease and mortality (Marmot \& Wilkinsson, 2006; Rajan et al, 2013). Still, scientists are debating the gradient linking socioeconomic status to a wide range of health outcomes (Rajan et al, 2013). Here, it was shown that the women came from one level of poverty and rose to the next level, from unemployment in the countryside to employment in the city, which gave them an income. This also gave them the possibility to adopt a new lifestyle and to buy "good food" in their understanding of it, i.e., fast, fried, processed food, and to appreciate and maintain a more sedentary lifestyle. The women in this study claimed that they could not afford to go to the "gym". This finding is supported by several scientists arguing that low family income is sometimes associated with worse health (Rajan et al, 2013 ). All the women commented that they now had money, but not enough to afford physical activity; they preferred using it on unhealthy food instead of traditional, healthy meals both for themselves and the family, because of the taste and also in order to save time. This exposed them, without their knowledge, to a higher risk of heart disease. According to the sense of Reasoned Action (Azjen \& Fishbein, 1980), the women in Kampala had simply adopted, and found meaning in, their new life situation, perceiving it as improved life quality. Still, during the interviews they showed an awakening interest in their lifestyles and the negative risks connected to that new life.

\section{Body Image and Culture}

In some parts of the African culture, women's beauty and attractiveness relate to a heavier body size as described before (Byrd-Brenner \& Murray, 2003 ), and confirmed in this study. Additionally, when the women, also in this study, entered the labor market, they could afford to eat well and that for them meant ready-made and fast foods. This diet also helped them to gain weight and acceptance in the eyes of others. This cultural perception emerged clearly in the interviews and has been supported by other scientists saying that African women who internalize the cultural standards have higher rates of eating disorder symptoms as compared to Caucasian women (Perez \& Joiner, 2003), the African women having a larger ideal body image, and being less likely to have a balanced diet to protect them from risks of heart disease. At the same time, some scientists indicate that this is changing in younger generations, towards a less heavy body ideal (Caldwell et al, 1997).

In this study one, woman stated that she used to have a "slender model-like body". This woman differed from the others, and she might be an early adopter of a changing African female body image.Having a big body (being heavy) was seen by the women living in Kampala as a documentation of an increase in life quality, of being healthy looking and beautiful and, as the Theory of Reasoned Action by Azden and Fishbein (1980) states, they adopted this view that "the significant others", i.e., partners, family and family-in law, had and incorporated that view in their lifestyle, and became all the more sedentary and overeating. This behavior was caused and strengthened by the transition from rural to urban life. All the women in this study had, thus, increased their weight. They fully accepted their heavier body image and desired to gain weight in order to feel accepted in society, beautiful, and attractive.

Women who have adapted to this kind of African culture tend to live their lives according to the wishes of others, as this culture places men, and even other family members, in charge of women's bodies, 
thus not allowing them to be in charge of their own bodies, as stated by Ajzden and Fishbein's theory (1980). They confirmed that African men were more willing to date women of a larger size, as elsewhere reported (Stevens et al, 1994)), and also that African women were less likely to diet and had no fear of weight gain; they rather felt pressure to gain weight and engage in overeating, as earlier research has also found (Perez \& Joiner, 2003).

\section{Lack of Knowledge}

In this study, it was also confirmed that the women, as they were getting older, were all in a very high risk zone for myocardial infarctions (MI) and related risk factors (Janzon et al, 2004), since they had all gained weight, and were inactive, as well as overconsumers of fatty foods. The women also revealed that they lived stressful lives. It has before been stated (The Theory of Health Believe by Ajzen\& Fishbein, 1980) that in order for a person to change livestyle, it is needed for them first to understand that the lifestyle the person has is a threat to that persons health. The women in this study were all unaware of their high risk of heart disease, by gaining weight and being inactive and about other risk factors related to that. Furthermore, they might represent a bulk of women belonging to the same age and socioeconomic group that, due to the economic shift in their society, have the same negative lifestyle and thereby have the same high risk of diabetes, hypertension, and myocardial infarction. It has been described before that women usually do not realise that heart disease can affect women, that more often they are afraid of getting cancer, and that heart disease is a "man's" disease" (Hart, 2005). The women in this study were also unaware of their high risk of that, in spite of having symptoms due to their overweight. The good news here was that the respondents themselves, after they had understood the relationship between inactivity, overweight, and risk of MI, asked for educational interventions in order to change their lifestyles and gain better health. They all showed an internal locus of control, i.e., they understood that only they themselves had the responsibility, and possibility, to take charge of their health, if they were helped to do so (Wallson \&Wallston, 1978).

\section{Caregivers Lack of or Commitment in Patients Health Information}

The respondents also had noticed, and showed now surprise to the fact, that hospital staff did not inform them about the negative health risks of overweight and inactivity, afterwards when they had understood the relationship between heart disease and its risk factors. They were weighed in health care centers and hospitals, but unfortunately did not get any health information or advice. This may suggest that the health professionals share the perception that "big is beautiful" in spite of their knowledge about the health risks associated with overweight. Furthermore, it is widely stated that if knowledge, support, and guidance are given by health professionals to the women, they will probably solve the problem themselves and make informed decisions individually or in groups (Beattie, 1991; Tengland, 2010).

\section{Public Health Recommendations}

From the findings of this study, the authorities of Uganda ought to start with public health strategies geared towards personal modification of lifestyles and behavior and also support health caregivers to be aware of their important role in the overall public health work, in order to curve the increasing rate of CVD (Akinboboye et al, 2003), so as to create public health measures targeted to this high risk group. Public health colleagues could be urged to search for inspiration by studying marketing strategies to "sell" healthy lifestyles, as food industries successfully do, sometimes by selling not always very healthy food. Television, local radio, women's magazines, schools, and churches could be possible partners for public health workers in order to raise knowledge about the health benefits of physical activity and healthy food, directed to the population 
The findings of this study may also be of interest to public health officers in countries with migrants who have their roots in similar African cultures, by increasing their understanding of cultural hindrances to public health measures and thus making it possible for them to suggest more appropriate lifestyle changes (Janzon et al, 2013).

\section{Conclusion}

Although the women acquired increased wealth, they decreased their health status, due to increased body weight and reduced physical activity, and they were unaware of the related risk of heart diseases, even if they experienced symptoms from their hearts. Interventions should be geared towards strategies based on empowerment theories, by empowering women, making them aware of the forces and structures formed by partners, family, and society, which make them uphold the traditional, unhealthy, and heavy female body image. Furthermore, caregivers ought to use their natural opportunities to inform about health risks and they ought to be supported by the authorities to see their role in overall public health strategies.

\section{Competing Interests}

The authors declare that they have no competing interests.

\section{Authors' Contributions}

EJ conceived of the study, participated in the design, drafted the manuscript. SN collected the data and wrote an early manuscript of the study. IB participated in its design and revised the manuscript critically. All authors read and approved the final manuscript.

\section{Acknowledgements}

The study was supported by "The Challenges of Migration", a scientific collaboration between Malmoe University and the city of Malmoe, and by Malmoe University.

To Katarina Graah-Hagelbäck for English language revision.

\section{References}

1. Ajzen, I. \& Fishbein, M. (1980). Understanding attitudes and predicting social behavior. Englewood Cliffs: Prentice Hall.

2. Akinboboye, O., Idris, 0., \& Akingkugbe, 0. (2003).Trends in coronary disease and associated risk factors in sub Saharan Africa. Journal of Human Hypertension, 17(6):381-7.

3. Bartky,W. T. (1990). Femininity and domination. Studies in the phenomenology of oppression. New York: Routledge.

4. Beattie, A. (1991). Knowledge and control in health promotion: A test case for social policy and social theory. In J. Gabe, M. Calman , \& M. Bury. (Eds), The sociology of health service. London: Routledge.

5. Burnard, P. (1991). A method of analyzing interview transcripts in qualitative research. Nurse Education Today, 11:461-465.

6. Buss, D. M. \& Schmitt, D. P. (2011). Evolutionary psychology and feminism. Sex Roles, 64:768-787.

7. Byrd-Bredbrenner, C. \& Murray, J. (2003). A comparison of the anthropometric measurements of idealized female body images in media directed to men, women, and mixed gender audiences. Topics in Clinical Nutrition,18:117-129.

8. Caldwell, B. M., Brownell, D. K., \& Wilfley, E. D. (1997). Relationship of weight, body dissatisfaction, and self-esteem in African and white female dieters. International Journal of Eating Disorders, 22:127-130.

9. Hart, P. (2005). Women's perceptions of coronary heart disease: an integrative review. Journal of Cardiovascular Nursing, 3:170-176.

10. Haskell, W. L., Lee, I. M., Pate, R. R., Powell, K. E., Blair, S. N., Franklin, B. A., Macera, C. A., Heath, G. W., Thompson, P. D., 
\& Bauman, A.2007 Physical Activity and Public Health. 39(8) 1423-34.

11. Janzon, E., Hedbland, B., Berglund, G., \& Engstrom, G. (2004). Tobacco and myocardial infarction in middle aged women. A study of factors modifying the risks. International Journal of Medical Science, 256: 111-118.

12. Kruger, H. S., Venter, S. C., \& Vorster, H. H. ( 2003). Physical inactivity as a risk factor for cardiovascular disease in communities undergoing rural to urban transition: the THUSA STUDY. Cardiovascular Journal of South Africa,

13. 14(1):16-23.

14. Kvale, S. \& Brinkman, S. (2009). Interviews learning the craft of qualitative research interviewing. Thousand Oaks, Ca: Sage.

15. Marmot, M. \& Wilkinson, R. G. (Eds.). (2006). Social Determinants of Health $\left(2^{\text {nd }}\right.$ ed.). Oxford: Oxford University Press,.

16. Martorell, R., Khan, K. L., Hughes, L. M.; \& Strawn, G. M. L. (2000). Obesity in women from developing countries. European Journal of Clinical Nutrition, 34:247-252.

17. Padgett, J. \& Biro, F. M. (2003). Different shapes in different cultures: Body dissatisfaction, overweight, and obesity in African-American and Caucasian females. Journal of Pediatric and Adolescent Gynecology, 16:349-354.

18. Perez, M. \& Joiner, E. (2003). Body image and disordered eating in black and white women. International Journal of Eating Disorders, 33(3):342-50.

19. Rajan, K., Kennedy, J., \& King, L. (2013). Is wealthier always healthier in poor countries? The health implications of income, inequality, poverty, and literacy in India. Social Science \& Medicine, 88:98-107.

20. Schenck-Gustafsson, K. (2009). Risk factors for cardiovascular disease in women. Maturitas, 63: 186-190.
21. Recommendation for Adults from the American College of Sports Medicine and the American Heart Association. (2007). Medicine \& HYPERLINK_ 39(8):1423-34.

22. Silverman, D. (2006). Interpreting qualitative data: methods for analyzing talk, text and interaction. London: Sage.

23. Stevens, J., Kumanyika, K.S., \& Keil, J.E. (1994). Attitudes towards Body Size and Dieting: Differences between Elderly Black and White Women. American Journal of Public Health, 84(8):1322-5.

24. Tengland, P.-A. (2010). Health promotion or disease prevention; a real difference for public health practices. Health Care Analysis, 18: 203-221

25. Uganda Bureau of statistics. (2006): The 2002 Uganda Population and Housing Census, Gender and Special Interest groups. Kampala, Uganda.

26. Uganda Ministry of Health: Health Sector Strategic Plan 11. [2005/2006 to 2009/2010, volume 1].

27. Coetzee, V. \& David, L. P. (2011). African and Caucasian body ideals in South Africa and United States. Eating Behavior, 12: $72-74$.

28. Wallston, K. A., Wallston, B. S., \& DeVellis, R. F. (1978). Locus of control and health: a review of the literature. Health Education Monographs. American Journal of Public Health, 6:107-17.

29. Wills, J. \& Naidoo, J. (2000). Health Promotion foundation for practice $\left(2^{\text {nd }}\right.$ ed.). London: Harcourt Publishers. 